\title{
Quality of object relations modifies the effectiveness of short- and long-term psychotherapy on self-concept
}

\author{
Olavi Lindfors ${ }^{1}$, Paul Knekt ${ }^{1,2}$, Esa Virtala ${ }^{1}$ \\ ${ }^{1}$ National Institute for Health and Welfare, Helsinki, Finland \\ ${ }^{2}$ Biomedicum Helsinki, Helsinki, Finland \\ Email: olavi.lindfors@,thl.fi
}

Received 26 May 2013; revised 28 June 2013; accepted 4 July 2013

Copyright (C) 2013 Olavi Lindfors et al. This is an open access article distributed under the Creative Commons Attribution License, which permits unrestricted use, distribution, and reproduction in any medium, provided the original work is properly cited.

\begin{abstract}
The quality of object relations affects interpersonal behaviour, but it is not known whether it modifies effectiveness on personality functioning in psychotherapies of different mode and length. In this study we estimated the modifying effect of the quality of object relations on the effect of solution-focused therapy (SFT) and short- and long-term psychodynamic psychotherapy (SPP and LPP) on self-concept. A total of 326 patients were assessed at baseline with the Quality of Object Relations Scale (QORS) and 4 times during a 3-year follow-up with the Structural Analysis of Social Behavior self-concept questionnaire, comprising altogether 10 scores on different aspects of self-concept pathology. The effectiveness of SFT, but not SPP, was significantly poorer in several domains $(5 / 10)$ of self-concept for patients with low QORS, i.e. those with less mature relational patterns, than for patients with high QORS, while the reversal occurred in some (3/10) self-concept domains in LPP. The results suggest that the quality of object relations has significance for treatment selection in therapies with different mode and length.
\end{abstract}

Keywords: Psychodynamic; Psychotherapy; Quality of Object Relations; Self-Concept; Solution-Focused

\section{INTRODUCTION}

Long-term changes in maladaptive and relatively enduring personality characteristics propose specific challenges beyond reduction of psychiatric symptoms for different psychotherapies and are often primary targets of therapy when personality pathology is involved [1]. The development of more benign internal self-representations, i.e. internalized mental models or schemas which guide behavior, affects and cognitions, according to the inter- personal and introject theory [2-4], is considered to represent sustained benefits of psychotherapies for individuals with affective and personality disorders [5]. The changes in the update of the Diagnostic and Statistical Manual of Mental Disorders (DSM-5) further highlight the importance of assessing the modification of personality disturbance on treatment outcome, and the respective treatment focuses on the disturbances of self and interpersonal relations [6].

Based on the Helsinki Psychotherapy Study [7], a randomized study on the effectiveness of two different modalities of short-term therapies, brief solution-focused (SFT) and transference-focused psychodynamic (SPP), and long-term psychodynamic psychotherapy (LPP) was conducted, we recently found that during the course and shortly after the end of the short-term therapies SFT and SPP, there was a more beneficial effect on self-concept than LPP [8]. However, at the 3-year follow-up after the start of the therapies, SFT, but not SPP, showed significantly less improved values than LPP, which in turn showed a more sustained growth in self-acceptance and a decreased tendency to punish and ignore oneself. Some support for greater self-concept changes, linked with somewhat longer therapy duration, has also been found in a study of brief to moderate length cognitive therapy [9], whereas in a study of two modalities of short-term psychodynamic psychotherapies with equal length, differences on a specific self-concept aspect, self-protect, appeared between therapies with different technique (transference focused vs. non-transference focused), modified by patient's motivation [10].

It is widely recognized that different types of patients are responsive to different types of psychotherapeutic treatments and therapy processes, and that patient factors may interact with therapy length, technique, and therapist characteristics $[5,11,12]$. To be clinically more applicable, findings on the general effectiveness need to be complemented further by studying the interaction effects 
of patient's global personality constellation and therapy length and modality on different types of therapy outcome. One of the most widely used factors in studying the modifying effects of patient's personality constellation, is the quality of object relations, which comprises the intrapsychic, developmental structures of self and object representations $[13,14]$. A person with a developmentally low level of object relations is characterized by a relatively instable self-structure, problematic interpersonal relations, immature defense mechanisms, and problems in regulating self-esteem and affects [14]. Accordingly, a favourable outcome in personality functioning has been suggested to benefit from the use of a transference-based technique with these patients, based on an extensive set of studies in SPP [e.g. 13,15-18]. However, there is a lack of knowledge whether the patient's quality of object relations is also relevant in non-psychodynamic short-term therapies, whether its effect differs in SPP and LPP, and along different self-concept dimensions.

In this study, we studied the possible effect modification of low vs. high quality of object relations on the outcome of SFT, SPP and LPP on self-concept during a 3-year follow-up.

\section{METHODS}

\subsection{Patients}

The patients, altogether 326 persons with mood or anxiety disorder were recruited for the Helsinki Psychotherapy Study between 1995 and 2000 [7]. Their age varied from 20 to 45 years, and a quarter of them were male. Most of the patients (82\%) had depressive disorder, $44 \%$ had anxiety disorder and $18 \%$ personality disorder. Patients with comorbid substance abuse disorder, severe somatic or psychiatric disorder, as well as those having received psychotherapy during the previous two years, were excluded. The patients were randomly assigned to either SFT (97 patients), SPP (101 patients) or LPP (128 patients) and monitored up to 3 years after the baseline. Written informed consent was received from all patients and the study was approved by the Helsinki University Hospital's ethics council

\subsection{Treatments and Therapists}

SFT is a brief resource-oriented therapeutic approach, based on a technique which helps clients change by constructing solutions [19]. The number of sessions in SFT was limited to 12 , over no more than 8 months. SPP is a transference-based, focal therapeutic approach which helps patients by exploring specific intrapsychic and interpersonal conflicts $[20,21]$. SPP was scheduled for 20 weekly sessions over 5 - 6 months. LPP is an open-ended, intensive, transference-based therapeutic approach which helps patients by exploring and working through a broad area of intrapsychic and interpersonal conflicts [22]. It was scheduled for 240 sessions, on average, and was provided usually twice a week up to 3 years. The therapies were carried out by qualified and experienced psychotherapists [23].

\subsection{Assessments}

Psychiatric diagnoses were assessed by interview, in accordance with the DSM-IV criteria [24]. Quality of object relations was also assessed by interview, using the Quality of Object Relations Scale (QORS) [25]. In scoring of the QORS, a total of 100 points was distributed to the five object relational levels (primitive, searching, controlling, triangular, mature), according to the estimated correspondence with prototypical descriptions of the scale and of the patient's object relations. The level scores were weighed (by 1, 3, 5, 7 and 9, respectively), and their sum divided by 100 . A dichotomized score $(\leq 5.0 ;>5.0)$ was used as an indicator of low and high QORS. Self-concept was measured at baseline and at 7, 12,24 , and 36 months after the baseline with the 36-item Structural Analysis of Social Behavior (SASB) introject questionnaire [26,27]. The primary outcome indicators were the two principal vector scores (ranging from -200 to +200 ), self-directed affiliation (AF) and self-directed autonomy (AU). In addition, eight cluster scale scores (self-free, self-affirm, self-love, self-protect, self-control, self-blame, self-attack, self-neglect) were calculated as a mean of the 4 to 5 items belonging to the specific cluster, the score values varying from 0 to 100 .

\subsection{Statistical Analysis}

The effectiveness analyses, described more thoroughly elsewhere $[8,23]$, were based on the intention-to-treat (ITT) design and, complemented by as-treated (AT) analyses and secondary analyses, where missing values were replaced by multiple imputation [28]. Linear mixed models [29] were used and model-adjusted mean levels of the outcome variables were calculated for the different measurement points [30]. The self-concept scores were used as the dependent variables in all analyses. The interaction between treatment group and QORS was analysed by a model which consisted main effects of time, treatment group, the difference between theoretical and realized date of measurement, and an interaction of time, treatment group and dichotomized QORS (low vs. high) group. Since no major differences were found between the different models, the results presented are based on the basic ITT model, based on the original data. The statistical analyses were carried out with the SAS software, version 9.1 [31]. 


\section{RESULTS}

The modifying effect of low vs. high QORS on the effectiveness of SFT, SPP and LPP was determined using the SASB self-concept scores, self-directed affiliation (AF), autonomy (AU), and eight cluster scores as outcome indicators. The QORS statistically significantly modified the outcome indicators in SFT and LPP but not in SPP (Table 1). In SFT, patients with low QORS benefitted less than patients with high QORS, indicated by poorer values in half $(5 / 10)$ of the self-concept scores (AU, self-attack, self-free, self-love, self-blame) during the course of the first 12 months of the follow-up. In the self-attack and the self-love scores, the difference between patients with low and high QORS were significant in SFT also at the 24 month follow-up, and in self-free also at the 36 month follow-up, indicated by a score difference of -8.15 (CI $=-14.3,-2.04)$. In LPP a reversed phenomenon was noted in three self-concept scores (AF, self-attack, self-love), indicating better outcomes, e.g. the AF score difference being $23.8(\mathrm{CI}=1.32,46.3)$ at the 12 month follow-up, for patients with low than high QORS, whereas thereafter no effect modification was found in any of the self-concept scores. The statistically significantly different change profiles in low vs. high QORS in SFT are illustrated by the self-free domain, where the modification was most extensive, in comparison to the respective differences in LPP (Figure 1).

\section{DISCUSSION}

As far as these authors know, this study is the first to compare the modifying effect of the quality of object relations on self-concept in psychotherapies with both different modality and length for patients with anxiety or depressive disorders. The study provided further evidence on the importance of patient's personality constellation, indicated by high vs. low QORS, when assessing the need for a specific type of short-term (SPP or SFT) or long-term (LPP) psychotherapy. In variance with results from earlier studies on short-term psychotherapies [32] we found a significant difference in the effectiveness of SPP and SFT on specific self-concept domains during a 3-year follow-up in a previous study [8]. In the present study we now demonstrated that QORS modified effectiveness only in SFT, being more effective for patients with high than with low QORS. This difference was notable in several self-concept scores, encompassing both the affiliation and the autonomy dimensions and highlighted in long-term differences in the domains of selfattack, self-love and self-free. We have also shown LPP to be generally more effective than SFT on self-concept [8]. In this study, it further emerged that LPP patients with low QORS benefitted more than those with high QORS during early phase of therapy in the dimension of affiliation and in the specific self-concept clusters selfattack and self-love.

The above findings of the differential modification of QORS may be explained by the SFT being a highly supportive, resource-oriented brief therapy [19] which might be more readily useful for patients with more benign developmental history and more balanced and mature internalized concept of oneself and others. For the pa-

Table 1. Baseline-adjusted significant ${ }^{\mathrm{a}}$ modification of low versus high ${ }^{\mathrm{b}}$ QORS on self-concept change scores in SFT and LPP from baseline to 7, 12, 24 and 36 months of follow-up.

\begin{tabular}{|c|c|c|c|c|c|}
\hline \multirow{3}{*}{$\begin{array}{c}\text { Outcome variable } \\
\text { (score) }\end{array}$} & \multirow{3}{*}{$\begin{array}{c}\text { Time } \\
\text { (month) }\end{array}$} & \multicolumn{4}{|c|}{ Mean level (low QORS) and score difference (95\% Confidence Interval) } \\
\hline & & SFT & & LPP & \\
\hline & & mean (SE) & Difference (low-high QORS) & mean $(\mathrm{SE})$ & Difference (low-high QORS) \\
\hline $\mathrm{AF}$ & 12 & $35.5(9.8)$ & n.s. & $38.4(9.1)$ & $23.8(1.32,46.3)$ \\
\hline $\mathrm{AU}$ & 7 & $-22.0(4.9)$ & $-14.8(-27.6,-2.11)$ & $-23.4(4.7)$ & n.s. \\
\hline \multirow[t]{3}{*}{ Self-attack } & 7 & $28.8(2.5)$ & $7.17(0.75,13.6)$ & $24.7(2.5)$ & n.s. \\
\hline & 12 & $25.9(2.7)$ & n.s. & $24.7(2.5)$ & $-6.68(-12.9,-0.46)$ \\
\hline & 24 & $30.1(3.1)$ & $8.48(0.52,16.4)$ & $22.4(2.7)$ & n.s. \\
\hline \multirow[t]{3}{*}{ Self-free } & 12 & $37.2(2.0)$ & $-6.03(-11.2,-0.90)$ & $38.4(1.9)$ & n.s. \\
\hline & 24 & $37.7(2.4)$ & $-7.62(-13.8,-1.49)$ & $37.5(2.0)$ & n.s. \\
\hline & 36 & $35.2(2.4)$ & $-8.15(-14.3,-2.04)$ & $39.5(2.1)$ & n.s. \\
\hline \multirow[t]{2}{*}{ Self-love } & 12 & $43.7(3.0)$ & $-10.1(-17.7,-2.49)$ & $48.3(2.7)$ & $8.46(1.72,15.2)$ \\
\hline & 24 & $42.6(3.9)$ & $-10.1(-20.0,-0.16)$ & $49.3(3.3)$ & n.s. \\
\hline Self-blame & 7 & $40.7(3.0)$ & $8.42(0.57,16.3)$ & $42.6(2.9)$ & n.s. \\
\hline
\end{tabular}

${ }^{a}$ The model consisted of main effects of time, treatment group, the difference between theoretical and realized date of measurement, and an interaction of time, treatment group and dichotomized QORS (low vs. high) group; $\mathrm{p}<0.05$; n.s., non-significant; ${ }^{\mathrm{b}}$ At baseline $46 \%$ of patients in SFT, $39 \%$ in SPP, and $38 \%$ in LPP were categorized as low QORS. LPP indicates long-term psychodynamic psychotherapy; SFT, solution-focused therapy; QORS, Quality of Object Relations Scale; AF, Affiliation; AU, Autonomy; no significant modification of QORS was noted in SPP (short-term psychodynamic psychotherapy) in any self-concept domains and neither in SFT and LPP in the self-affirm, self-protect, self-control and self-neglect scores at any measurement point. 


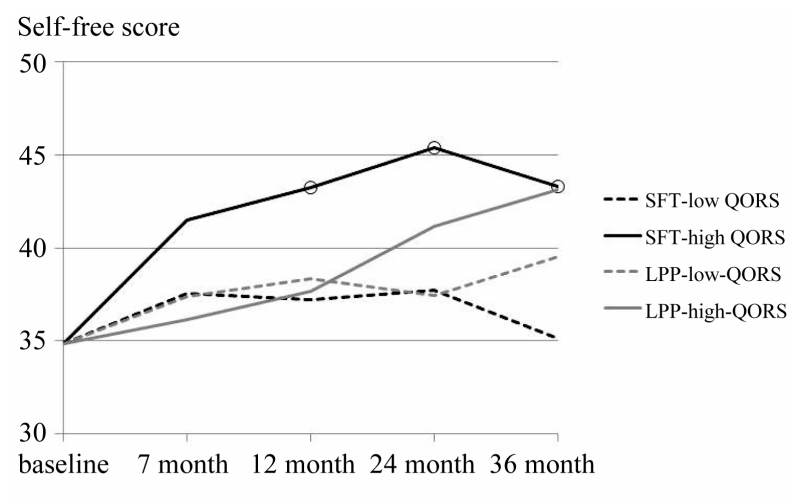

O indicates statistically significant difference between high and low QORS in SFT

Figure 1. Interaction of therapy group and low vs. high QORS in SFT and LPP on changes in the SASB self-free score, baseline adjusted.

tients with high QORS, the approach of SFT could be used like an empowering coaching in facing the need to reconstruct the self in more positive terms [33]. Instead, the patients with low QORS, i.e. those who are more vulnerable due to lesser trust in oneself and in others, more previous disappointments, ruptures and primitive defenses in relationships, might not be able to modify their more instable sense of self in a brief therapy with a highly supportive technique which does not include exploration of pathological self and object representations within the therapeutic relationship.

The fact that patients with low QORS fared somewhat better than those with high QORS in LPP during the first year of treatment, can be understood as being related to the interplay between the nature of the treatment process and the nature of the patients' relational problems. Patients with low QORS are expected to be both more prone to repeat openly their problematic internal relationships with the therapist, as well as to be able to benefit more from re-experiencing and being sensitively confronted by these patterns in the transference than patients with high QORS [13,14]. This may lead to more pronounced and faster increase in self-esteem in moderate to long-term therapy, possibly mediated by a stronger alliance $[11,16]$, as there are plenty of opportunities to explore maladjusted relational patterns. In contrast, patients with high QORS (and, accordingly, with more benign self-concept), may find that the confronting and exploratory technique of LPP does not primarily enhance the positive elements of their self-concept. Instead, it offers the possibility to explore more fully potentially conflictuous self-experiences, as they manifest in recurring patterns in behavior, feelings, and relationships during the treatment. Accordingly, in the long run, patients with high QORS also seem to be able to benefit significantly from LPP.

In variance with the results on SFT and LPP, no modifying effect of QORS was found in the short-term, ex- plorative SPP, unlike in some previous studies where high QORS was related to more favorable outcome in the respective type of SPP [18]. It has also been repeatedly, and mostly consistently [e.g., 13,15-17], shown that patients with low QORS seem to benefit, by decreased symptoms and improved personality functioning, more from explorative SPP with a pronounced transferencefocus than without it. However, unlike in all the previous studies focused on transference-interpretations, the therapists in this study were not instructed to use primarily or exclusively transference-based interpretations, since the therapies were carried out along the general guidelines presented by Malan [20] and Sifneos [21]. Accordingly, the lack of interaction between QORS and treatment effect on self-concept in SPP may signify therapists' modification of the technique in relation to patient's characteristics.

The findings of this study have clinical implications concerning pre-treatment assessment of patients' suitability for different types of short-term or long-term psychotherapy, as well as for developing more optimally tailored treatments for patients, by acknowledging the level of their object relations. The significance of QORS as a differential modifier of outcome in SFT and LPP suggests that it can provide the clinician important preparatory information on the challenges of the planned therapy, as the QORS level tends to activate specific relational patterns with the therapist [34]. Supportive, brief therapies might thus be sufficient for self-concept change when the patient's object relational patterns are relatively mature, while optimal therapy for patients with low QORS seems to require a modality which encompasses working with complex issues within the therapeutic relationship, like in the different psychotherapies for patients with personality disorder $[1,16,35]$.

The advantages of this study include the use of a relatively large sample of patients, treated by trained therapists and evaluated by a thorough baseline assessment and repeated outcome measurements. However, although adjustments due to withdrawal, compliance and potential confounding were made, it is still possible that there may be some unidentified bias. Findings on the outcome indicators were generally consistent and relevant for core psychopathology, although in four self-concept scores (self-affirm, self-protect, self-control, self-neglect) no interaction was found in any therapy group. Further study is needed to examine which self-concept domains are most important for sustained recovery beyond the 3 -year follow-up. In addition, although the therapists were trained and committed to provide the specific therapy modality according to its general guidelines, it is not known to what extent they may have used therapy-specific or non-specific techniques when treating patients with high vs. low QORS. However, this also allows more 
potential for generalizing the findings, albeit requires further investigation on the issue.

\section{CONCLUSION}

In conclusion, the modifying effect of QORS was studied for the first time in both psychodynamic and non-psychodynamic brief therapies and in LPP. QORS appeared to modify differently the outcome in LPP and SFT on several aspects of self-concept, but not in SPP. In SFT, smaller long-term treatment effects on self-concept were noted in patients with low QORS than in patients with high QORS, while in LPP especially low QORS patients benefited more at early phases of the treatment. The results suggest that QORS has significance for treatment selection in therapies with different mode and length.

\section{ACKNOWLEDGEMENTS}

This study was supported financially by the Academy of Finland (grant nr. 138876).

\section{REFERENCES}

[1] Bedics, J.A., Atkins, D.C., Comtois, K.A. and Linehan, M.M. (2012) Treatment differences in the therapeutic relationship and introject during a 2-year randomized controlled trial of dialectic behavior therapy versus nonbehavioral psychotherapy experts for borderline personality disorder. Journal of Consulting and Clinical Psychology, 80, 66-77. http://dx.doi.org/10.1037/a0026113

[2] Altwood, G.E. and Stolorow, R.D. (1980) Psychoanalytic concepts and the representational world. Psychoanalytic Contemporary Thought, 3, 267-290.

[3] Benjamin, L.S. (1974) Structural analysis of social behavior. Psychological Review, 81, 392-425. http://dx.doi.org/10.1037/h0037024

[4] Benjamin, L.S. (1994) SASB: A bridge between personality theory and clinical psychology. Psychological Inquiry, 5, 273-316. http://dx.doi.org/10.1207/s15327965pli0504_1

[5] Blatt, S.J., Zuroff, D.C., Hawley, L.L. and Auerbach, J.S. (2010) Predictors of sustained therapeutic change. Psychotherapy Research, 20, 37-54. http://dx.doi.org/10.1080/10503300903121080

[6] Wright, A.G.C., Pincus, A.L., Hopwood, C.J., Thomas, K.M., Markon, K.E. and Krueger, R.F. (2012). An interpersonal analysis of pathological personality traits in DSM-5. Assessment, 19, 263-275. http://dx.doi.org/10.1177/1073191112446657

[7] Knekt, P., Lindfors, O., Härkänen, T., Välikoski, M., Virtala, E., Laaksonen, M.A., Marttunen, M., Kaipainen, M., Renlund, C, and the Helsinki Psychotherapy Study Group (2008) Randomized trial on the effectiveness of long- and short-term psychodynamic psychotherapy and solution-focused therapy on psychiatric symptoms during a 3-year follow-up. Psychological Medicine, 38, 689-703. http://dx.doi.org/10.1017/S003329170700164X
[8] Lindfors, O., Knekt, P., Virtala, E., Laaksonen, M.A. and the Helsinki Psychotherapy Study Group (2012) The effectiveness of solution-focused therapy and short- and long-term psychodynamic psychotherapy on self-concept during a 3-year follow-up. Journal of Nervous and Mental Disease, 200, 946-953. http://dx.doi.org/10.1097/NMD.0b013e3182718c6b

[9] Vittengl, J.R., Clark, L.A. and Jarrett, R.B. (2004) Selfdirected affiliation and autonomy across acute and continuation phase cognitive therapy for recurrent depression. Journal of Personality Assessment, 83, 235-247. http://dx.doi.org/10.1207/s15327752jpa8303 07

[10] Marble, A., Hoglend, P. and Ulberg, R. (2011) Change in self-protection and symptoms after dynamic psychotherapy: The influence of pretreatment motivation. Journal of Clinical Psychology, 67, 355-367. http://dx.doi.org/10.1002/jclp.20771

[11] Hersoug, A.G., Hoglend, P., Gabbard, G.O. and Lorentzen, S. (2012) The combined predictive effect of patient characteristics and alliance on long-term dynamic and interpersonal functioning after dynamic psychotherapy. Clinical Psychology \& Psychotherapy, 20, 297-307. http://dx.doi.org/10.1002/cpp.1770

[12] Laaksonen, M.A., Knekt, P. and Lindfors, O. (2012) Psychological predictors of the recovery from mood or anxiety disorder in short-term and long-term psychotherapy during a 3-year follow-up. Psychiatry Research, 208, 162173. http://dx.doi.org/10.1016/j.psychres.2012.09.053

[13] Hoglend, P., Johansson, P., Marble, A., Bogwald, K.-P. and Amlo, S. (2007) Moderators of the effects of transference interpretations in brief dynamic psychotherapy. Psychotherapy Research, 17, 160-171. http://dx.doi.org/10.1080/10503300701194206

[14] Piper, W.E., Ogrodniczuk, J.S. and Joyce, A.S. (2004) Quality of object relations as a moderator of the relationship between pattern of alliance and outcome in shortterm individual psychotherapy. Journal of Personality Assessment, 83, 345-356. http://dx.doi.org/10.1207/s15327752jpa8303 15

[15] Hoglend, P., Hersoug, A.G., Bogwald, K.-P., Amlo, S., Marble, A., Sorbye, O., Rossberg, J.I., Ulberg, R., Gabbard, G.O. and Crits-Christoph, P. (2011) Effects of transference work in the context of therapeutic alliance and quality of object relations. Journal of Consulting and Clinical Psychology, 79, 697-706. http://dx.doi.org/10.1037/a0024863

[16] Hoglend, P., Dahl, H.-S., Hersoug, A.G., Lorentzen, S. and Perry, J.C. (2011) Long-term effects of transference interpretation in dynamic psychotherapy of personality disorders. European Psychiatry, 26, 419-424. http://dx.doi.org/10.1016/j.eurpsy.2010.05.006

[17] Piper, W.E., Azim, H.F., Joyce, A.S. and McCallum, M. (1991) Transference interpretations, therapeutic alliance, and outcome in short-term individual psychotherapy. Archives of General Psychiatry, 48, 946-953. http://dx.doi.org/10.1001/archpsyc.1991.01810340078010

[18] Piper, W.E., Joyce, A.S., McCallum, M. and Azim, H.A. (1998) Interpretive and supportive forms of psychotherapy and patient personality variables. Journal of Consult- 
ing and Clinical Psychology, 66, 558-567. http://dx.doi.org/10.1037/0022-006X.66.3.558

[19] de Shazer, S., Berg, I.K., Lipchik, E., Nunnally, E., Molnar, A., Gingerich, W. and Weiner-Davis, M. (1986) Brief therapy: Focused solution development. Family Process, 25, 207-221. http://dx.doi.org/10.1111/j.1545-5300.1986.00207.x

[20] Malan, D.H. (1976) The frontier of brief psychotherapy: An example of the convergence of research and clinical practice. Plenum Medical Book, New York. http://dx.doi.org/10.1007/978-1-4684-2220-7

[21] Sifneos, P.E. (1978) Short-term anxiety provoking psychotherapy. In: Davanloo, H., Ed., Short-Term Dynamic Psychotherapy, Spectrum, New York, 35-42.

[22] Gabbard, G.O. (2004) Long-term psychodynamic psychotherapy: A basic text. American Psychiatric Press, Washington DC.

[23] Knekt, P. and Lindfors, O. (2004) A randomized trial of the effect of four forms of psychotherapy on depressive and anxiety disorders: Design, methods, and results on the effectiveness of short-term psychodynamic psychotherapy and solution-focused therapy during a one-year follow-up. Social Insurance Institution, Helsinki, 15-112. http://www.thl.fi/hps

[24] American Psychiatric Association (1994) Diagnostic and statistical manual of mental disorders: DSM-IV. American Psychiatric Association, Washington DC.

[25] Azim, H.F., Piper, W.E., Segal, P.M., Nixon, G.W. and Duncan, S.C. (1991) The quality of object relations scale. Bulletin of the Menninger Clinic, 55, 323-343.

[26] Benjamin, L.S. (1996) A clinician-friendly version of the interpersonal circumplex: Structural analysis of social behavior (SASB). Journal of Personality Assessment, 66,
248-266. http://dx.doi.org/10.1207/s15327752jpa6602 5

[27] Benjamin, L.S. (2000) SASB user's manual. University of Utah, Salt Lake City.

[28] Rubin, D.B. (1987) Multiple imputation for nonresponse in surveys. John Wiley, New York. http://dx.doi.org/10.1002/9780470316696

[29] Verbeke, G. and Molenberghs, G. (1997) Linear mixed models in practice: An SAS-oriented approach. Springer, New York. http://dx.doi.org/10.1007/978-1-4612-2294-1

[30] Lee, J. (1981) Covariance adjustment of rates based on the multiple logistic regression model. Journal of Chronic Diseases, 34, 415-426. http://dx.doi.org/10.1016/0021-9681(81)90040-0

[31] SAS Institute Inc. (2007) SAS/STAT 9.1 User's guide. SAS Institute Inc., Cary.

[32] Abbass, A., Town, J. and Driessen, E. (2011) The efficacy of short-term psychodynamic psychotherapy for depressive disorders with comorbid personality disorder. Psychiatry, 74, 58-71. http://dx.doi.org/10.1521/psyc.2011.74.1.58

[33] Kärkkäinen, H. (2001) Construing the self-Identity processes in resource-oriented therapy. Rehabilitation Foundation, Helsinki, Research Reports 68.

[34] Diguer, L., Gamache, D. and Laverdière, O. (2012) Development and initial validity of the object relations rating scale. Psychotherapy Research, 22, 402-416. http://dx.doi.org/10.1080/10503307.2012.662606

[35] Critchfield, K.L. (2012) Tailoring common treatment principles to fit individual personalities. Journal of Personality Disorders, 26, 108-125. http://dx.doi.org/10.1521/pedi.2012.26.1.108 